This item was submitted to Loughborough's Research Repository by the author.

Items in Figshare are protected by copyright, with all rights reserved, unless otherwise indicated.

\title{
Noise-controlled signal transmission in a multithread semiconductor neuron
}

PLEASE CITE THE PUBLISHED VERSION

http://dx.doi.org/10.1103/PhysRevLett.102.226802

\section{PUBLISHER}

(C) American Physical Society

VERSION

VoR (Version of Record)

LICENCE

CC BY-NC-ND 4.0

\section{REPOSITORY RECORD}

Samardak, A., A. Nogaret, Natalia B. Janson, Alexander G. Balanov, I. Farrer, and David A. Ritchie. 2019. "Noise-controlled Signal Transmission in a Multithread Semiconductor Neuron". figshare.

https://hdl.handle.net/2134/12736. 
This item was submitted to Loughborough's Institutional Repository (https://dspace.lboro.ac.uk/) by the author and is made available under the following Creative Commons Licence conditions.

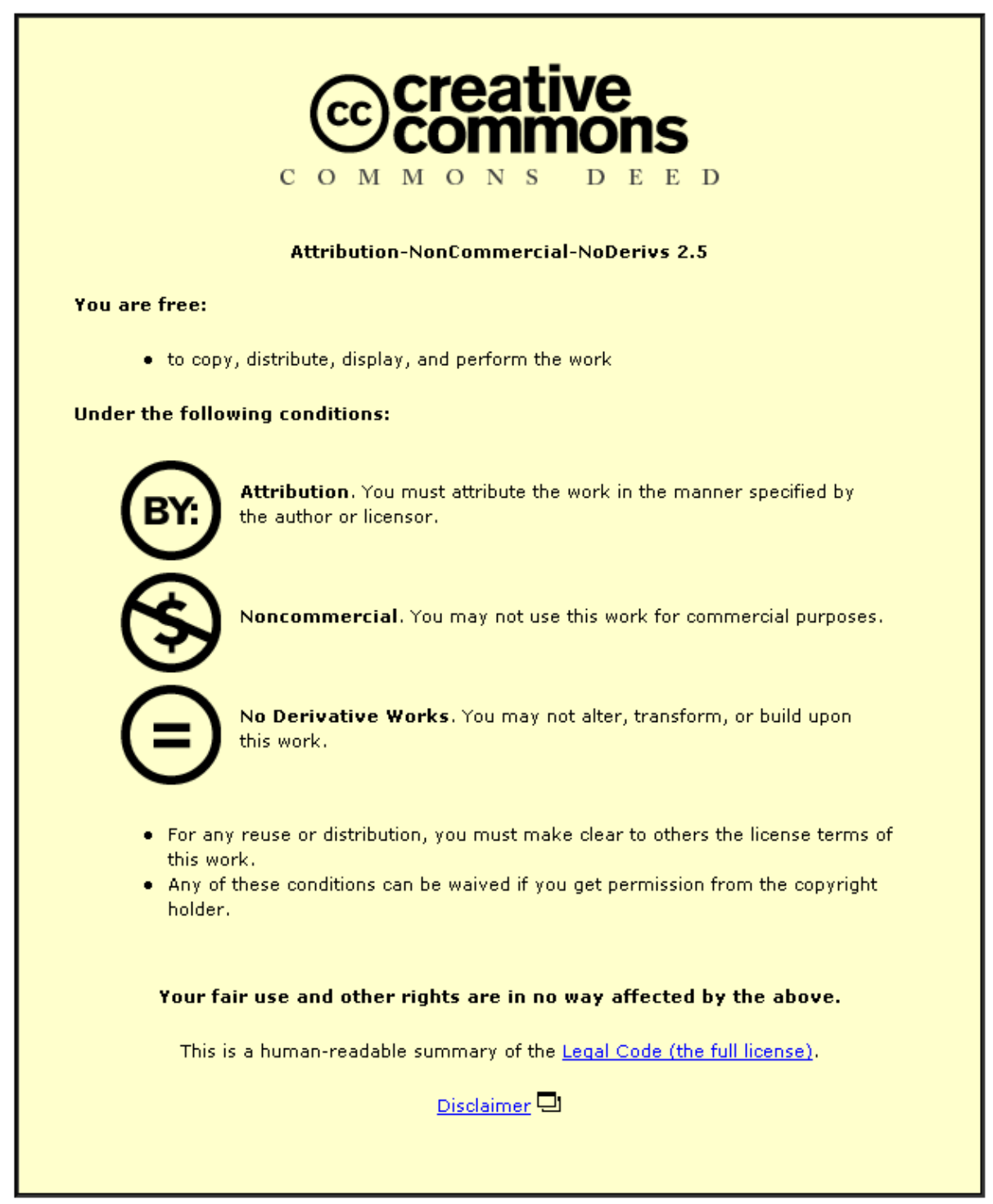

For the full text of this licence, please go to: http://creativecommons.org/licenses/by-nc-nd/2.5/ 


\title{
Noise-Controlled Signal Transmission in a Multithread Semiconductor Neuron
}

\author{
A. Samardak and A. Nogaret* \\ Department of Physics, University of Bath, Claverton Down, Bath BA2 7AY, United Kingdom \\ N. B. Janson \\ School of Mathematics, University of Loughborough, Loughborough LE11 3TU, United Kingdom \\ A. G. Balanov \\ Department of Physics, University of Loughborough, Loughborough LE11 3TU, United Kingdom \\ I. Farrer and D. A. Ritchie \\ Cavendish Laboratory, University of Cambridge, Cambridge CB3 OHE, United Kingdom \\ (Received 11 January 2009; published 5 June 2009)
}

\begin{abstract}
We report on stochastic effects in a new class of semiconductor structures that accurately imitate the electrical activity of biological neurons. In these devices, electrons and holes play the role of $\mathrm{K}^{+}$and $\mathrm{Na}^{+}$ ions that give the action potentials in real neurons. The structure propagates and delays electrical pulses via a web of spatially distributed transmission lines. We study the transmission of a periodic signal through a noisy semiconductor neuron. Using experimental data and a theoretical model we demonstrate that depending on the noise level and the amplitude of the useful signal, transmission is enhanced by a variety of nonlinear phenomena, such as stochastic resonance, coherence resonance, and stochastic synchronization.
\end{abstract}

DOI: 10.1103/PhysRevLett.102.226802

PACS numbers: 85.35.- p, 05.45.-a, 87.85.-d

It remains a challenging task to create an artificial structure [1,2] which would reproduce the essential properties of real neurons and, in particular, the spatiotemporal charge redistribution stimulated by the exchange of ion currents. Considerable progress in this direction was recently reported in [3-5] where semiconductor $p-n$ wires were shown to provide a suitable medium for the transmission of electrical pulses in the manner of biological neurons. However, an essential feature of real neurons is their ability to function in a stochastic environment [6,7], and, in particular, to exploit the power of random noise to enhance the propagation of useful signals along their axons [8,9]. It is important to reproduce this property in hardware in order to create a realistic neuronlike structure.

In this Letter we report on the performance of a semiconductor neuron that is subjected to the combined stimulation of a random Gaussian noise and a periodic perturbation applied to its inputs. We have experimentally found that the proposed device is able to demonstrate the whole spectrum of nontrivial stochastic phenomena $[10,11]$, some of which were observed earlier in biological neurons: coherence resonance [12], stochastic resonance [13-15] and stochastic synchronization [16]. In all of the above effects the increase in the strength of random noise from zero to some moderate value, counterintuitively, leads to more regular oscillations in the system. We identify an optimal level of noise at which spiking is most regular, while either too much or too little noise results in less ordered motion in the system. We study the transition between coherence and stochastic resonances to demonstrate that this transition is accompanied by the phenomenon of stochastic synchronization. We propose a theoretical model which describes the performance of the semiconductor neuron in very good agreement with experimental data. The neuron is well suited to making networks capable of learning sequences of interspike intervals without supervision [17], of simulating neocortical architectures $[18,19]$ and probing the effects of time delayed coupling on neural synchronization $[20,21]$.

The key elements of real neurons are the dendrites, soma, and axon. Dendrites and axons are nerve fibers that transmit electrical signals to and from the soma. The soma collects (sums and integrates) the electrical inputs from individual dendrites. Crucially, soma and axon are connected by an axon hillock-an element that produces a travelling spike in the axon if the total signal arriving at the soma is larger than a certain threshold value. The axon hillock thus behaves as a threshold amplifier.

Artificial "neurons" were synthesized from GaAs/AlAs layers grown by molecular beam epitaxy according to the following sequence: $n$ type: $5 \times 10^{17} \mathrm{~cm}^{-3}(80 \mathrm{~nm}) / p$ type: $5 \times 10^{17} \mathrm{~cm}^{-3}(60 \mathrm{~nm}) / \mathrm{AlAs} / n^{+}$type: $6 \times$ $10^{18} \mathrm{~cm}^{-3}(40 \mathrm{~nm}) / p^{+}$type $4 \times 10^{19} \mathrm{~cm}^{-3}(500 \mathrm{~nm}) /$ GaAs buffer. Through a combination of selective etching and lithography described elsewhere [3,4], we obtain the free standing neuronlike structure shown in Fig. 1(a). p-n wires form a web of microtransmission lines ("dendrites") which meet at the active center of the structure (soma-axon hillock). 
(a)
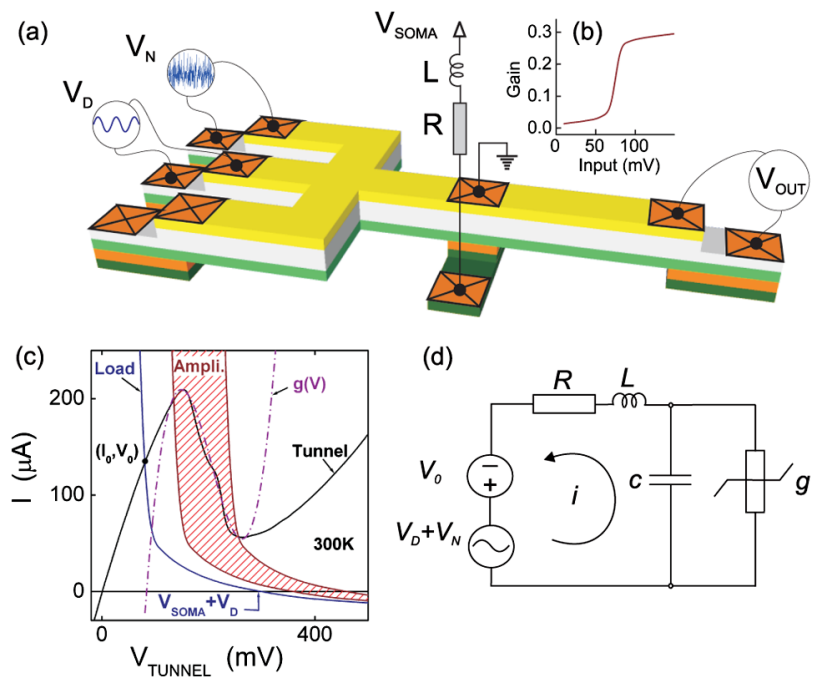

FIG. 1 (color online). (a) Solid state neuron excited by a sinusoidal drive of amplitude $V_{D}$ and a white noise with standard deviation (rms voltage) $V_{N}$. Depolarization waves emanating from input contacts diffuse along the $p-n$ wire and interfere in the central region (soma) before reaching the output. The $p^{+}-n^{+}$ tunnel layers are the pillars supporting the free standing $p-n$ lines. (b) Sigmoid gain curve of the tunnel amplifier. (c) $I-V$ characteristics of the tunnel device ( $\mathrm{N}$-shaped) and the load line including circuit elements $R=0 \Omega, L=10 \mathrm{mH}, V_{\text {soma }}=$ $290 \mathrm{mV}$ and the $p-n$ wire. (d) Equivalent circuit of the artificial soma.

The depletion region of the $p-n$ wire is physically equivalent to the nerve membrane. It supports the propagation of depolarization waves in much the same way as nerve fibers do propagate action potentials because both transmission lines obey the same diffusion equation [5]. The diffusion process integrates the inputs which arrive at the "soma" delayed and attenuated. The attenuation factor is 8.8 (35) in the short (long) dendrite [4]. If the sum of incoming signals is larger than the threshold voltage $V_{\text {th }}=$ $80 \mathrm{mV}$, the soma emits a depolarization wave into the "axon." Threshold amplification is implemented by a $p^{+}-n^{+}$quantum tunnelling amplifier whose sigmoid gain curve is shown in Fig. 1(b). The gain curve was obtained by applying rectangular pulses and measuring the height of the output pulses $V_{\text {out }}$ as a function of the amplitude of input pulses. The gain is the ratio of output to input pulse heights.

The external circuit pictured in Fig. 1(a) is used to control the excitable regime of the soma. Under experimental conditions, the tunnel characteristics was biased as shown in Fig. 1(c). The hatched region corresponds to selfoscillations of the soma. The series resistance was $R=$ $0 \Omega$ [22] and $V_{\text {soma }}$ was set below the onset of selfoscillations when the operating point $\left(I_{0}, V_{0}\right)$ is at the edge of the negative differential resistance region. A small perturbation through the $p-n$ wire in this case activates a large spike before the soma returns to equilibrium. The property of excitability is therefore achieved through the positive feedback of the tunnel layers on the $p$ - $n$ wire.
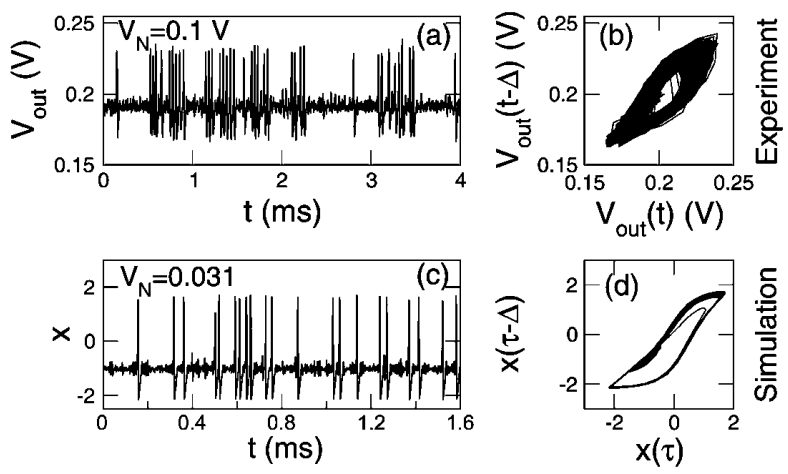

FIG. 2. Sequence of output spikes for $V_{D}=0$ : (a) experimental, (c) calculated. Phase portraits: (b) experimental $\Delta=$ $1.6 \mu \mathrm{s}$, (d) theoretical $\Delta=0.075 \sqrt{L C}$.

Here, we set $V_{\text {soma }}=290 \mathrm{mV}$ to obtain the threshold $V_{\mathrm{th}}=80 \mathrm{mV}$ described above. The tunnel element has capacitance $C=1.6 \mathrm{nF}$ [3], $L=10 \mathrm{mH}$, and the frequency of self-oscillations of soma is $30 \mathrm{kHz}$. The equivalent circuit of the soma is pictured in Fig. 1(d). Figure 2(a) shows a typical spiking sequence output by the neuron when noise only was applied. A phase portrait showing the stochastic limit cycle, reconstructed from this sequence by delay embedding, is shown in (b). This behavior is also found by simulating Eqs. (2)—see (c),(d).

The property of excitability enables the neuron to transmit a subthreshold periodic signal with the help of noise: the energy of noise is used to amplify the periodic signal, and the amplification is the largest at some optimal noise intensity. This phenomenon is known as stochastic resonance [23]. We have applied a sinusoidal voltage to one

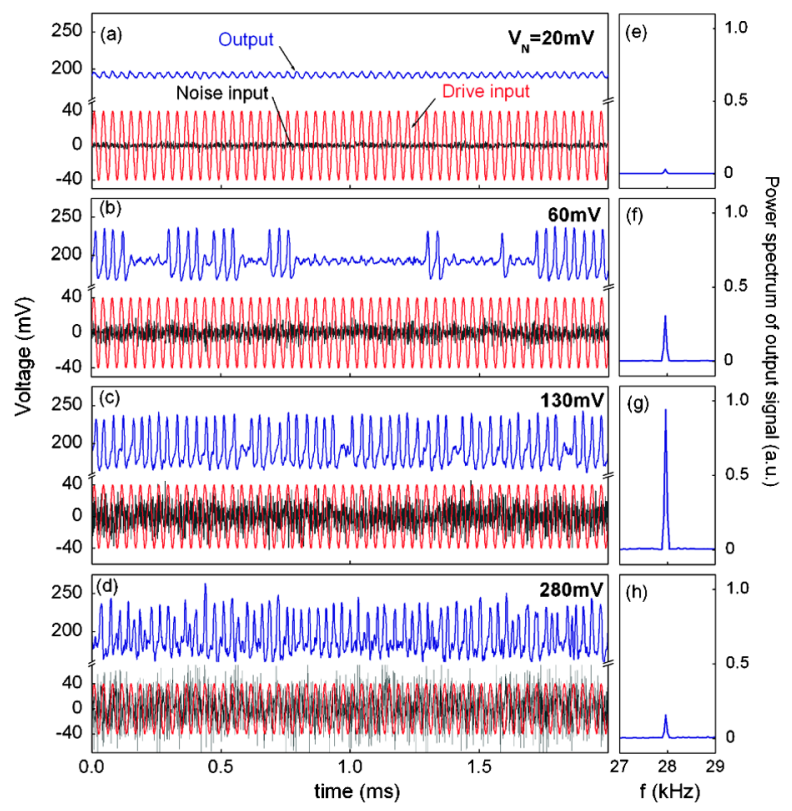

FIG. 3 (color online). (a)-(d) Sequences of spiking events at four noise intensities across stochastic resonance. For clarity, the noise is shown $\times 10^{-1}$. (e)-(h) Power spectral densities of the output signal. The peak passes through a maximum at $V_{N}=$ $130 \mathrm{mV}$. 
dendrite, a digitally synthesized Gaussian white noise to another, and measured the output waveform with a DAQ acquisition card. Drive frequencies $f_{D}=1 \mathrm{kHz}-30 \mathrm{kHz}$ were investigated for signal amplitudes ranging from subthreshold to superthreshold. Experimental outcomes were similar when uniformly distributed noise was used instead.

To find the conditions of optimum amplification, we increase $V_{N}$ from $20 \mathrm{mV}$ to $280 \mathrm{mV}$ in Fig. 3. With no or vanishing noise [panel (a)], the $40 \mathrm{mV}$ subthreshold signal decays passively through the structure. Spiking bursts appear and become more frequent as noise increases. The output is closest to being periodic at $V_{N}=$ $130 \mathrm{mV}$ [panel (c)]. Further increases in noise level restore irregular spiking patterns [panel (d)]. The power spectrum, in panels (e)-(h), shows that the amplified frequency is the $28 \mathrm{kHz}$ frequency of the input drive, and that optimum amplification occurs at $V_{N}=130 \mathrm{mV}$, thus demonstrating stochastic resonance.

To quantify the amplification of a periodic signal by noise, we plot the characteristics of output oscillations in Fig. 4. The output spectrum was decomposed into the noise background and the peak at $f_{D}$. Three integrals were calculated: the integral over the full spectrum (total power $P_{\text {tot }}$ ), over the noise background (power of noise $P_{\text {noi }}$ ), and over the peak [power of periodic component $P_{\text {per }}$ (a),(d)]. In (b),(e) the mean spiking frequency is shown. Finally, to enable comparison with the case when periodic driving is absent, the regularity of spiking was characterized by the standard deviation of interspike intervals normalized by the mean spiking period, $R_{T}=\sqrt{\operatorname{Var}(T)} /\langle T\rangle(\mathrm{c}),(\mathrm{f})$.

At $V_{D}=0 \mathrm{mV}$ [solid line in (c)], the noise-induced spiking is closest to periodic at an optimal $V_{N}=210 \mathrm{mV}$ (largest value of $1 / R_{T}$ ), and this is associated with coherence resonance (CR) [24,25]. The spiking frequency is $30 \mathrm{kHz}$ [solid line in (b)], slightly above the driving frequency, $f_{D}=28 \mathrm{kHz}$.

At $V_{D}=40 \mathrm{mV}$, the coherence peak occurs at slightly lower noise while the mean frequency develops a small plateau at $f_{D}=28 \mathrm{kHz}$ [full circles in (b)]. $P_{\text {tot }}$ and $P_{\text {noi }}$ grow monotonically with $V_{N}$ at almost the same rate at both small and large noise. At intermediate noise, $P_{\text {tot }}$ increases faster than $P_{\text {noi }}$ allowing $P_{\text {per }}$ to pass through the stochastic resonance maximum described above [(a) full circles].

At $V_{D}=70 \mathrm{mV}$, the coherence peak has shifted further to lower noise [(c) open circles] however the situation is somewhat different. At small noise $\left(V_{N}<14 \mathrm{mV}\right)$, the neuron is totally silent, but as soon as spiking starts at $V_{N} \approx 14 \mathrm{mV}$, the frequency is close to that of external driving for a large range of noise intensities [(b), open circles], which is evidence of stochastic synchronization $[15,26] . P_{\text {per }}$ [(a) full circles] jumps to its maximum value at $V_{N} \approx 14 \mathrm{mV}$, and has an extended plateau. At $V_{N}>$ $210 \mathrm{mV}, f_{\exp }$ increases monotonically with noise. This noise driven pulsing mode is robust and almost independent of $V_{D}$ [panel (b)].

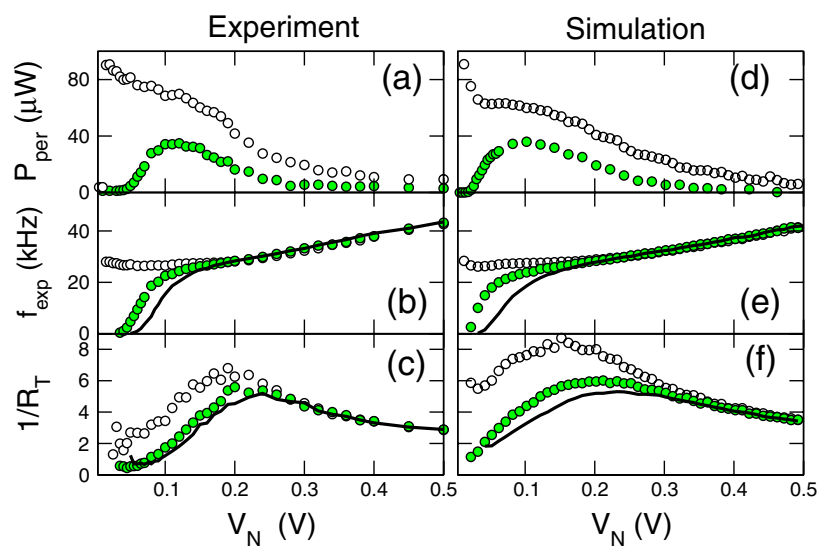

FIG. 4 (color online). Characteristics of the neuron output as a function of noise at three amplitudes of the drive signal: $V_{D}=$ $70 \mathrm{mV}$ (open symbols), $40 \mathrm{mV}$ (full symbols), 0 (solid line). (a), (c) Power of periodic component at $f_{D}=28 \mathrm{kHz}$; (b),(e) mean spiking frequency; (c),(f) coherence of interspike intervals. The simulated frequency (power) was converted from dimensionless to experimental units by multiplying with $3.4 /(|g| L)(340 \mu \mathrm{W}$.)

At suprathreshold amplitudes, $V_{D}>80 \mathrm{mV}$, oscillations are no longer noise-induced. Spiking becomes periodic at frequency $f_{D}$, and noise smears it. This is shown in Fig. 5 (a) where $P_{\text {per }}$ decays to zero as $V_{N}$ increases. The spiking frequency $f_{\text {exp }}$ is mapped in panel (b).

A model of the neuron was constructed from first principles. We assume that the dendrites and axon attenuate signals, but otherwise do not transform them. The crucial part of the neuron is the soma with axon hillock, whose equivalent circuit is given in Fig. 1(d). Kirchhoff's equations of this circuit are:

$$
\frac{d V}{d t}=\frac{1}{C}(i-g(V)), \quad \frac{d i}{d t}=-\frac{1}{L}\left(V+R i+V_{a}+V_{\text {soma }}\right),
$$
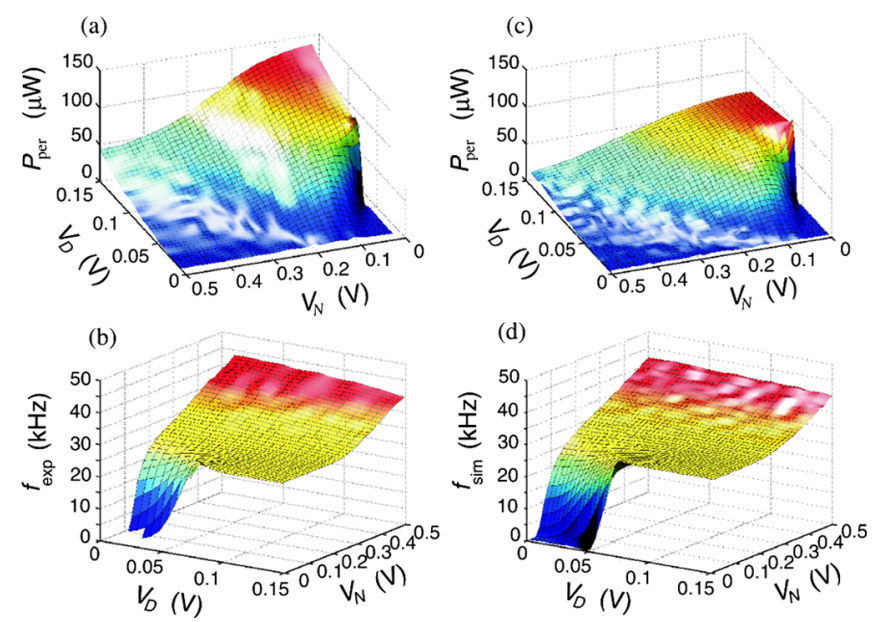

FIG. 5 (color online). (a),(c) Power of periodic component; (b),(d) mean spiking frequency as a function of drive and noise amplitudes. Left: experimental data; right: simulation with Eqs. (2). 
where $V$ is the voltage across the tunnel element, $i$ is the current through the soma and $g(V)=114 \mu \mathrm{A}[((V-$ $\left.206 \mathrm{mV}) / 57 \mathrm{mV})^{3} / 3-(V-206 \mathrm{mV}) / 57 \mathrm{mV}\right]+132 \mu \mathrm{A}$ is the cubic polynomial approximating the negative differential region of the tunnel characteristics in Fig. 1(c). $V_{a}=$ $\left[V_{D} \sin \left(2 \pi f_{D} t\right)+V_{N} \xi_{2}(t)\right]$ is the time dependent voltage (periodic drive + noise). After making the following change of variables,

$$
\begin{gathered}
x=\frac{V-206 \mathrm{mV}}{57 \mathrm{mV}}, \quad y=\frac{132 \mu \mathrm{A}-i}{114 \mu \mathrm{A}}, \\
\tau=\frac{1}{|g| L} t, \quad \epsilon=\frac{C}{|g|^{2} L}, \\
\alpha=\frac{\left(V_{\mathrm{th}} / 8.8\right)+57 \mathrm{mV}}{57 \mathrm{mV}}, \quad V_{D}^{\prime}=\frac{V_{D}}{8.8 \times 57 \mathrm{mV}}, \\
V_{N}^{\prime}=\frac{V_{N}}{35 \times 57 \mathrm{mV}}, \quad f_{D}^{\prime}=f_{D}|g| L,
\end{gathered}
$$

and with account of $R=0 \Omega$, Eqs. (1) are transformed into the dimensionless FitzHugh-Nagumo equations [27]:

$$
\left\{\begin{array}{l}
\epsilon \dot{x}=\tilde{g}(x)-y+V_{N}^{\prime} \xi_{1}(\tau), \\
\dot{y}=x+\alpha+V_{D}^{\prime} \sin \left(2 \pi f_{D}^{\prime} \tau\right)+V_{N}^{\prime} \xi_{2}(\tau),
\end{array}\right.
$$

where $x$ is the membrane potential, $y$ is the recovery variable and $|g|=2 \mathrm{mS}(114 \mu \mathrm{A} / 57 \mathrm{mV})$ is the negative differential conductance of the tunnel element [22]. Unlike in the classical model, here the external input $\left[V_{D}^{\prime} \sin \left(2 \pi f_{D}^{\prime} \tau\right)+V_{N}^{\prime} \xi_{2}(\tau)\right]$ is applied through the voltage in the second equation, rather than through the current in the first equation. Also, we introduce the uncorrelated sources of noise $\xi_{1,2}$ into both equations to take a better account of noises in the real devices.

Parameters $\epsilon$ and $\alpha$ were obtained accurately by fitting the data in the range $[0 ; 0.2 \mathrm{~V}]$ for $V_{D}^{\prime}$ and $[0 ; 0.6 \mathrm{~V}]$ for $V_{N}^{\prime}$. The method is accurate because the neuron output is quite sensitive to the variation of $\alpha$. Figures 2, 4, and 5 show a good agreement between the experiment and the model. $\epsilon$ and $\alpha$ were also estimated by inserting the circuit parameters into the transformation equations. The fitted (estimated) values are $\epsilon=0.1$ (0.04) and $\alpha=1.02$ (1.16). The transformation formulas allow converting $V_{N}^{\prime}, V_{D}^{\prime}$ in quantitative agreement with $V_{N}$ and $V_{D}$ whereas the simulated $f_{D}^{\prime}$ is factor 3.4 smaller when converted into SI units. This discrepancy is due to the approximation of the $I-V$ curve by a 3rd order polynomial $g(V)$ which breaks down far from the negative differential resistance region.

We have also found that the noise dependence in Fig. 4(c) at $V_{N}>210 \mathrm{mV}$ and Fig. 5(b), can be accounted for by considering an extra noise source, $\xi_{1}(t)$, in addition to the intentionally applied noise $\xi_{2}(t)$. The extra noise source is believed to arise from the current surges in the soma circuit which regenerate pulses. The passage of such current through the Ohmic contact resistance at the center of Fig. 1(a) generates shot noise. Noise sources $\xi_{1}(t)$ and $\xi_{2}(t)$ are thus uncorrelated.
In summary, we have constructed a spatially delocalized neuron which provides understanding of the interplay between stochastic resonance, coherence resonance and stochastic synchronization in a controlled environment. The neuron provides direct feedback on mathematical models and is useful as a versatile building block of future complex networks.

We thank S. Taylor for graphics. A. S. and A.N. acknowledge the support of Leverhulme Trust under grant $(\mathrm{F} / 0035 / \mathrm{N})$ and N.B. J. acknowledges the support of the EPSRC (UK).

*A.R.Nogaret@bath.ac.uk

[1] J. Nagumo, S. Arimoto, and S. Yoshizawa, Proc. IRE 50, 2061 (1962).

[2] D. Coon, S. Ma, and A. Perera, Phys. Rev. Lett. 58, 1139 (1987).

[3] A. Samardak et al., New J. Phys. 10, 083010 (2008).

[4] A. Samardak et al., Appl. Phys. Lett. 91, 073502 (2007).

[5] A. Nogaret et al., Europhys. Lett. 68, 874 (2004).

[6] L. Ricciardi and L. Sacerdote, Biol. Cybern. 35, 1 (1979).

[7] D. Kamran, H. Lester, and C. Koch, J. Neurosci. 24, 9723 (2004).

[8] J. Douglass et al., Nature (London) 365, 337 (1993).

[9] W. Stacey and D. Durand, J. Neurophysiol. 86, 1104 (2001).

[10] L. Gammaitoni, P. Hänggi, P. Jung, and F. Marchesoni, Rev. Mod. Phys. 70, 223 (1998).

[11] V. Anishchenko, V. Astakhov, A. Neiman, T. Vadivasova, and L. Schimansky-Geier, Nonlinear Dynamics of Chaotic and Stochastic Systems (Springer, Berlin, 2002).

[12] H. Gu et al., NeuroReport 13, 1657 (2002).

[13] J. Levin and J. Miller, Nature (London) 380, 165 (1996).

[14] T. Mori and S. Kai, Phys. Rev. Lett. 88, 218101 (2002).

[15] A. Neiman, A. Silchenko, V. Anischenko, and L. Shimansky-Geier, Phys. Rev. E 58, 7118 (1998).

[16] A. Neiman and D. Russel, Phys. Rev. Lett. 88, 138103 (2002).

[17] H. Abarbanel and S. Talathi, Phys. Rev. Lett. 96, 148104 (2006).

[18] H. Makram, Nat. Neurosci. 7, 153 (2006).

[19] T. M. Morse, Bioinformatics and Biol. Insights 2, 259 (2008).

[20] M. Dhamala, V. Jirsa, and M. M. Ding, Phys. Rev. Lett. 92, 074104 (2004).

[21] G. Franceschini, S. Bose, and E. Schöll, Phys. Rev. E 60, 5426 (1999).

[22] Quantum tunneling amplification requires $|g R|<1$. Since $|g|=2 m S, R<500 \Omega$ and we choose $R=0$.

[23] R. Benzi et al., Tellus 34, 10 (1982).

[24] H. Gang, T. Ditzinger, C. Ning, and H. Haken, Phys. Rev. Lett. 71, 807 (1993).

[25] A. Pikovsky and J. Kurths, Phys. Rev. Lett. 78, 775 (1997).

[26] B. Shulgin, A. Neiman, and V. Anishchenko, Phys. Rev. Lett. 75, 4157 (1995).

[27] R. FitzHugh, Biophys. J. 2, 11 (1962). 